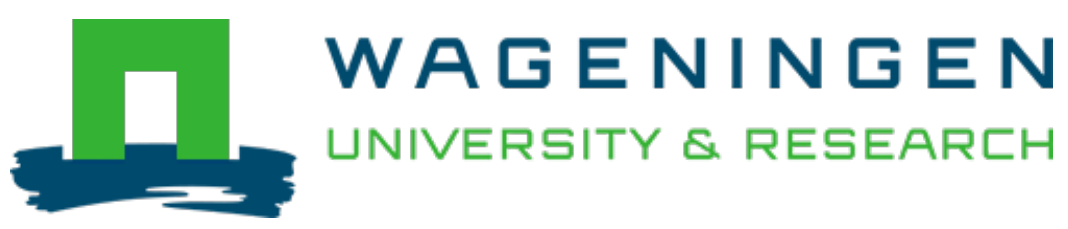

\author{
The impact of selected strains of probiotic bacteria on metabolite formation in set \\ yoghurt \\ International Dairy Journal \\ Settachaimongkon, S.; Nout, M.J.R.; Antunes Fernandes, E.C.; Hooijdonk, A.C.M.; Zwietering, M.H. et al \\ https://doi.org/10.1016/j.idairyj.2014.04.002
}

This publication is made publicly available in the institutional repository of Wageningen University and Research, under the terms of article $25 \mathrm{fa}$ of the Dutch Copyright Act, also known as the Amendment Taverne. This has been done with explicit consent by the author.

Article 25 fa states that the author of a short scientific work funded either wholly or partially by Dutch public funds is entitled to make that work publicly available for no consideration following a reasonable period of time after the work was first published, provided that clear reference is made to the source of the first publication of the work.

This publication is distributed under The Association of Universities in the Netherlands (VSNU) 'Article $25 \mathrm{fa}$ implementation' project. In this project research outputs of researchers employed by Dutch Universities that comply with the legal requirements of Article $25 \mathrm{fa}$ of the Dutch Copyright Act are distributed online and free of cost or other barriers in institutional repositories. Research outputs are distributed six months after their first online publication in the original published version and with proper attribution to the source of the original publication.

You are permitted to download and use the publication for personal purposes. All rights remain with the author(s) and / or copyright owner(s) of this work. Any use of the publication or parts of it other than authorised under article $25 \mathrm{fa}$ of the Dutch Copyright act is prohibited. Wageningen University \& Research and the author(s) of this publication shall not be held responsible or liable for any damages resulting from your (re)use of this publication.

For questions regarding the public availability of this publication please contact openscience.library@wur.nl 


\title{
The impact of selected strains of probiotic bacteria on metabolite formation in set yoghurt
}

\author{
Sarn Settachaimongkon ${ }^{\mathrm{a}, \mathrm{b}, \mathrm{c}}$, M. J. Robert Nout ${ }^{\mathrm{b}}$, Elsa C. Antunes Fernandes ${ }^{\mathrm{a}}$, \\ Toon C.M. van Hooijdonk ${ }^{\mathrm{a}}$, Marcel H. Zwietering ${ }^{\mathrm{b}}$, Eddy J. Smid ${ }^{\mathrm{b}}$, \\ Hein J.F. van Valenberg ${ }^{\mathrm{a}, *}$ \\ a Dairy Science and Technology Group, Chair of Food Quality and Design, Wageningen University, P.O. Box 17, 6700 AA Wageningen, The Netherlands \\ ${ }^{\mathrm{b}}$ Laboratory of Food Microbiology, Wageningen University, P.O. Box 17, 6700 AA Wageningen, The Netherlands \\ ${ }^{\mathrm{c}}$ Department of Food Technology, Faculty of Science, Chulalongkorn University, Bangkok 10330, Thailand
}

\section{A R T I C L E I N F O}

\section{Article history:}

Received 9 November 2013

Received in revised form

8 April 2014

Accepted 10 April 2014

Available online 26 April 2014

\begin{abstract}
A B S T R A C T
The influence of Lactobacillus rhamnosus GG and Bifidobacterium animalis subsp. lactis BB12 in cofermentation with traditional starters on metabolite formation in set yoghurt was evaluated. Microbial activity during fermentation and refrigerated storage was investigated by monitoring bacterial population dynamics, milk acidification and overall changes in yoghurt metabolite profiles. A complementary metabolomics approach using solid-phase microextraction-gas chromatography/mass spectrometry and ${ }^{1} \mathrm{H}$ nuclear magnetic resonance resulted in the identification of 37 volatile and 43 non-volatile metabolites, respectively. Results demonstrated that the two probiotic strains did not influence acidity and the key-aroma volatile metabolites of set yoghurt. However, a contribution by the presence of $L$. rhamnosus GG on the non-volatile metabolite profile of yoghurt was specifically noticed during storage. Multivariate analysis allowed yoghurts fermented by different starter combinations and different durations of storage to be differentiated according to their metabolite profiles. This provides new insights regarding the impact of probiotics on the metabolome of yoghurt.
\end{abstract}

(ㄷ) 2014 Elsevier Ltd. All rights reserved.

\section{Introduction}

Yoghurt is a dairy product obtained by lactic acid fermentation, the production of which can be regarded as one of the oldest methods of preserving milk (Tamime \& Robinson, 2007). According to the Codex standard for fermented milks (Codex, 2003), yoghurt is specifically characterised by the presence of symbiotic cultures of two lactic acid bacteria (LAB), i.e., Streptococcus thermophilus and Lactobacillus delbrueckii subsp. bulgaricus. Successful manufacture of yoghurt relies on the mutual interaction, also called protocooperation, between these two species. Proto-cooperation is not only linked with lactic acid production but also with the formation of distinctive flavour and texture characteristics of the product (Courtin \& Rul, 2004). Nowadays, societal interest in healthy food products has contributed to the development of functional dairy products that potentially provide health benefits in addition to the nutrients they contain (Shiby \& Mishra, 2013).

\footnotetext{
* Corresponding author. Tel.: +31 317482284.

E-mail address: hein.vanvalenberg@wur.nl (H.J.F. van Valenberg).
}

An example of a functional type of dairy products is yoghurt with probiotic incorporation. These bacteria (i.e., probiotics) are defined as live microorganisms that, when administered in adequate amounts, confer a health benefit on the host (FAO/WHO, 2002). To ensure their health-promoting effects, a probiotic product should contain at least $10^{6} \mathrm{cfu}^{-1}$ of viable probiotic cells throughout the entire shelf-life (Vasiljevic \& Shah, 2008). Most commercial probiotics incorporated in dairy products are strains belonging to the genera Lactobacillus and Bifidobacterium (LourensHattingh \& Viljoen, 2001). Members of these two genera have a long history of safe use in the manufacture of fermented food products and can be found as a part of the normal microbiota in the human gastrointestinal tract (Shah, 2000). Despite high numbers of studies on strain selection, safety concerns, health-promoting properties and technological approaches to improve the viability of probiotics in fermented dairy products (Mohammadi, Sohrabvandi, \& Mohammad-Mortazavian, 2012; Shiby \& Mishra, 2013), the actual metabolic activity of probiotics grown or suspended in milk is not yet fully understood (Plessas, Bosnea, Alexopoulos, \& Bezirtzoglou, 2012). This information is important, since the organic acids and volatiles formed by these non-starter 
bacteria may directly contribute to the organoleptic quality of product (Østlie, Treimo, \& Narvhus, 2005).

Metabolomics is recognised as an effective tool for investigating the overall chemical composition of complex biological systems including food matrices (Herrero, Simo, Garcia-Canas, Ibanez, \& Cifuentes, 2012). The application of mass spectrometry (MS)-based and nuclear magnetic resonance (NMR)-based techniques have been shown to be very effective for determining a wide range of metabolites in liquid milk (Boudonck, Mitchell, Wulff, \& Ryals, 2009; Klein et al., 2010; Lu et al., 2013) and fermented dairy products (Consonni \& Cagliani, 2008; Piras et al., 2013; Rodrigues et al., 2011). However, the number of publications focussing on metabolomics as a tool to investigate the activity of probiotics in fermented dairy products is rather limited (Mozzi, Ortiz, Bleckwedel, De Vuyst, \& Pescuma, 2013; Rodrigues et al., 2011).

A previous study by the authors successfully demonstrated the influence of different proteolytic activity of starter bacteria, i.e., S. thermophilus and L. delbrueckii subsp. bulgaricus on biochemical characteristics of set yoghurt from a metabolomics perspective (Settachaimongkon et al., 2014). As a consequence, the objective of this study was to evaluate the impact of two commercial probiotics, Lactobacillus rhamnosus GG and Bifidobacterium animalis subsp. lactis BB12, in co-fermentation with traditional starters, on metabolite formation in set yoghurt. Changes in bacterial population, decrease of $\mathrm{pH}$ and increase in lactic acid concentration were monitored during set yoghurt fermentation and storage. Biochemical changes associated with bacterial metabolism were characterised in terms of volatile and non-volatile polar metabolite profiles using headspace solid phase microextraction-gas chromatography/MS (SPME-GC/MS) and ${ }^{1} \mathrm{H}$ NMR techniques. Finally, metabolite profiles of different yoghurt samples were statistically compared using multivariate analysis.

\section{Materials and methods}

\subsection{Yoghurt starters and probiotic strains}

Frozen direct-vat-inoculation pellets of S. thermophilus C44, L. delbrueckii subsp. bulgaricus C49 (CSK Food Enrichment, Ede, The Netherlands) and B. animalis subsp. lactis BB12 (Chr. Hansen, Hørsholm, Denmark) were stored at $-45{ }^{\circ} \mathrm{C}$ and defrosted at ambient temperature $\left(20 \pm 3^{\circ} \mathrm{C}\right)$ for $15 \mathrm{~min}$ before use. A freezedried culture of L. rhamnosus GG (ATCC 53103) was propagated in MRS broth and stored as a $20 \%$ (v/v) glycerol stock-culture at $-80{ }^{\circ} \mathrm{C}$. The cultures were refreshed in MRS broth (Merck, Darmstadt, Germany) at $37^{\circ} \mathrm{C}$ for $24 \mathrm{~h}$ under anaerobic conditions (Anoxomat ${ }^{\mathrm{TM}}$ Mart $^{\circledR}$ Microbiology, Drachten, The Netherlands). Then, the cells were collected by centrifugation at $4000 \times g$ for $15 \mathrm{~min}$ at $4{ }^{\circ} \mathrm{C}$, washed twice using peptone-physiological-salt solution (Tritium microbiology, Eindhoven, The Netherlands) and finally resuspended in milk before use. Set yoghurts were fermented with three different types of starter combinations: (i) traditional yoghurt starters $(\mathrm{Y})$ consisting equal numbers of S. thermophilus C44 and L. delbrueckii subsp. bulgaricus C49, (ii) coculture of yoghurt starters with $L$. rhamnosus GG (Y-LGG) and (iii) co-culture of yoghurt starters with $B$. animalis subsp. lactis BB12 (Y$\mathrm{BB} 12)$. The combination of the two yoghurt starters and probiotic strain were inoculated each at $10^{6} \mathrm{cfu} \mathrm{g}^{-1}$ at the starting point of fermentation (ratio 1:1:1).

\subsection{Set yoghurt fermentation}

Nilac skimmed milk powder (NIZO Food Research, Ede, The Netherlands) was reconstituted and pasteurised according to the method described by Settachaimongkon et al. (2014). After inoculation, set yoghurt fermentation was carried out in a water bath at $42{ }^{\circ} \mathrm{C}$ for $4 \mathrm{~h}$. Then, yoghurts were placed in a cold chamber $\left(4 \pm 2{ }^{\circ} \mathrm{C}\right)$ for 28 days of storage. Samples were taken hourly during fermentation and weekly during storage. The enumeration of viable bacteria and determination of acidity were carried out immediately after sampling. For ${ }^{1} \mathrm{H}$ NMR, the samples were stored at $-20{ }^{\circ} \mathrm{C}$ until analysis. The fermentation was performed in three replicates for each type of starter combination.

\subsection{Enumeration of viable bacteria}

Viable counts of $S$. thermophilus were determined on S. thermophilus agar after aerobic incubation at $37{ }^{\circ} \mathrm{C}$ for $24 \mathrm{~h}$ (Ashraf \& Shah, 2011). Viable counts of L. delbrueckii subsp. bulgaricus in yoghurt fermented with (i) Y, (ii) Y-BB12 and (iii) Y-LGG were determined on: (i) MRS agar pH 5.70 (Merck) after anaerobic, (ii) modified atmosphere $\left(6 \% \mathrm{O}_{2}, 7 \% \mathrm{CO}_{2}\right.$ ) incubation (Anoxomat ${ }^{\mathrm{TM}}$ Mart ${ }^{\circledR}$ Microbiology) at $37{ }^{\circ} \mathrm{C}$ for $48 \mathrm{~h}$ (modified from Ashraf \& Shah, 2011), and (iii) MRS agar pH 5.7 supplemented with $20 \mathrm{mg} \mathrm{L}^{-1}$ ciprofloxacin (Sigma-Aldrich, Steinheim, Germany) after anaerobic incubation at $37^{\circ} \mathrm{C}$ for $48 \mathrm{~h}$ (tested in this study). Viable counts of $L$. rhamnosus GG were determined on MRS agar supplemented with $50 \mathrm{mg} \mathrm{L}^{-1}$ vancomycin (Merck) after anaerobic incubation at $37^{\circ} \mathrm{C}$ for $48 \mathrm{~h}$ (Tharmaraj \& Shah, 2003). Viable counts of $B$. animalis subsp. lactis BB12 were determined on MRS agar supplemented with $0.5 \mathrm{~g} \mathrm{~L}^{-1}$ cysteine-HCl (Merck) and $50 \mathrm{mg} \mathrm{L}^{-1}$ mupirocin (LGC Standards, Middlesex, UK) after anaerobic incubation at $37{ }^{\circ} \mathrm{C}$ for $48 \mathrm{~h}$ (Ashraf \& Shah, 2011).

\subsection{Determination of acidification profile}

Production of acid during set yoghurt fermentation and storage was expressed by changes in $\mathrm{pH}$ and increases in titratable acidity as described previously (Settachaimongkon et al., 2014).

\subsection{Analysis of volatile metabolites by headspace SPME-GC/MS}

For headspace SPME-GC/MS analysis, a model scenario of set yoghurt fermentation was carried out directly in GC vials (Settachaimongkon et al., 2014). The fermentation was performed in three replicates for each type of starter combination. Samples were analysed at the end of fermentation ( $4 \mathrm{~h}$ ) and every two weeks during storage ( $14 \mathrm{~d}$ and $28 \mathrm{~d}$ ). Extraction and determination of volatile compounds by headspace SPME-GC/MS were performed according to the method previously described by Settachaimongkon et al. (2014). This method was based on the method developed by Hettinga, van Valenberg, Lam, and van Hooijdonk (2008). Volatile metabolites were identified using AMDIS software (NIST, Gaithersburg, MD, USA) referred to NIST/ EPA/NIH database and library provided by Hettinga, van Valenberg, Lam, and van Hooijdonk (2009). Specific retention time and $m / z$ model were used for automated peak integration in the XCalibur software package (Thermo Scientific, Austin, TX, USA).

\subsection{Analysis of non-volatile polar metabolites by ${ }^{1} \mathrm{H} N M R$ spectroscopy}

For ${ }^{1} \mathrm{H}$ NMR analysis, two replicates of samples at the end of fermentation $(4 \mathrm{~h})$ and one replicate of stored samples (14 $\mathrm{d}$ and $28 \mathrm{~d}$ ) were prepared according to the method previously described (Settachaimongkon et al., 2014). NOESY 1D- ${ }^{1} \mathrm{H}$ NMR measurements were performed at $300 \mathrm{~K}$ in a $600 \mathrm{MHz}$ NMR spectrometer (Bruker, Rheinstetten, Germany) operated under full automation, with similar parameters as described by Lu et al. (2013). The ${ }^{1} \mathrm{H}$ NMR spectra were baseline-corrected, phase-corrected, aligned and 
calibrated based on the internal standard (TSP) peak. For each spectrum, chemical shift $(\delta)$ across the range of $0.00-10.00 \mathrm{ppm}$ was segmented (binning) with an interval of $0.02 \mathrm{ppm}$ (Settachaimongkon et al., 2014). The signal intensity in each bin was integrated and expressed in arbitrary units using AMIX software (Bruker). Metabolite labels were assigned to the bins by means of Chenomx NMR suite 7.5 library (Chenomx Inc., Alberta, Canada) and from the list of metabolites identified by Settachaimongkon et al. (2014). For unlabelled bins, significant variables were selected based on one-way ANOVA at 95\% confidence level.

\subsection{Statistical analysis}

One-way analysis of variance (ANOVA) with multiple comparisons by Tukey's test were performed using the IBM SPSS statistics package version 19 (SPSS Inc., Chicago, IL, USA). A probability of $P<0.05$ was considered statistically significant. Metabolomics data from GC/MS and ${ }^{1} \mathrm{H}$ NMR were normalised by median-centering and $\log _{2}$-scaling before multivariate statistical analysis (Settachaimongkon et al., 2014). Principal component analysis (PCA), heat-map visualisation and Pearson's correlation-based hierarchical cluster analysis (HCA) were performed using MultiExperiment Viewer (MeV) version 4.8 (www.tm4.org/mev/).

\section{Results and discussion}

\subsection{Bacterial growth and survival}

In the samples fermented with standard yoghurt starters $(\mathrm{Y}$; Fig. 1A), S. thermophilus developed rapidly during the early stage of fermentation, especially during $0-3 \mathrm{~h}$, while $L$. delbrueckii subsp. bulgaricus remained in lag-phase for at least one hour. Similar growth patterns of the two species of traditional yoghurt starters have been well documented (Courtin \& Rul, 2004; Tamime \& Robinson, 2007). At the end of fermentation, the viable counts of S. thermophilus and L. delbrueckii subsp. bulgaricus increased by 2.3 and $2.0 \log$ units to reach an average value of $8.7 \pm 0.2$ and $8.3 \pm 0.3 \log \mathrm{cfu} \mathrm{g}^{-1}$, respectively. The viable counts of the two species remained nearly stable (above $8.0 \log \mathrm{cfu}^{-1}$ ) throughout the 28-day storage period. The high viability of $S$. thermophilus has been well recognised. However, many authors have found that the viability of $L$. delbrueckii subsp. bulgaricus was strain-dependent and a loss of viability down to $1.5 \mathrm{log}$ units during refrigerated storage has been reported (Donkor, Henriksson, Vasiljevic, \& Shah, 2006; Oliveira, Sodini, Remeuf, Tissier, \& Corrieu, 2002; Saccaro, Tamime, Pilleggi, \& Oliveira, 2009).

In co-culture with L. rhamnosus GG (Y-LGG; Fig. 1B) or B. animalis subsp. lactis BB12 (Y-BB12; Fig. 1C), yoghurt starters showed similar growth and viability pattern as found in the samples fermented without probiotics. These results suggest no obvious interference from the addition of $L$. rhamnosus GG or B. animalis subsp. lactis BB12 on the growth and viability of yoghurt starters. It can be noted that $S$. thermophilus predominates the microbial population in all tested culture combinations. This observation confirms reports of other researchers (Oliveira et al., 2002; Saccaro et al., 2009). On the other hand, the two probiotic strains exhibited different growth patterns and varied in their viability during storage. The viable counts of L. rhamnosus GG and B. animalis subsp. lactis BB12 inoculated individually in milk remained stable during incubation at $42{ }^{\circ} \mathrm{C}$ for $4 \mathrm{~h}$ (data not shown).

The limited capacity of $L$. rhamnosus GG to grow in milk is explained by the lack of ability to ferment lactose. The weak proteolytic activity along with a requirement for low redox potential, explain the poor growth of $B$. animalis subsp. lactis BB12 in milk
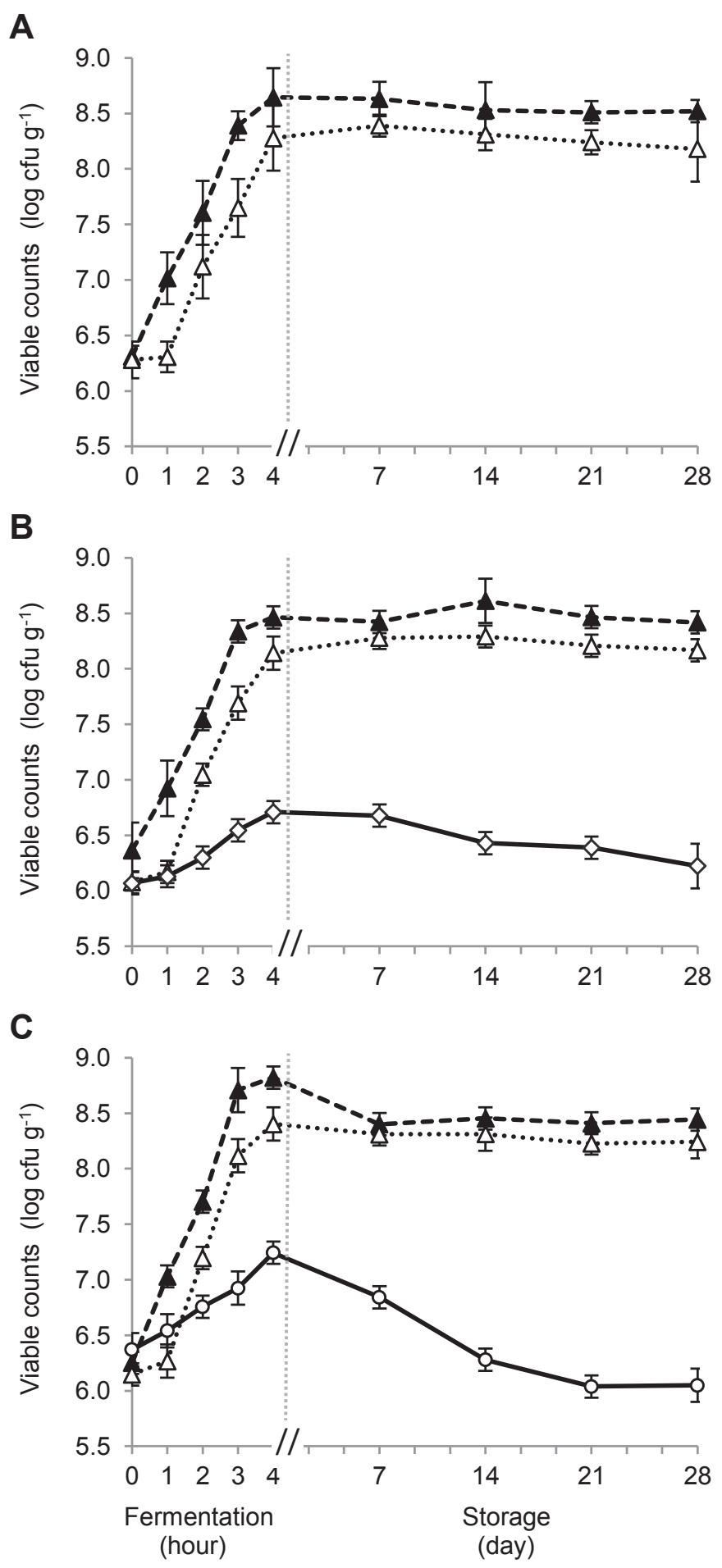

Fig. 1. Changes in viable bacteria counts during set-yoghurt fermentation ( $4 \mathrm{~h}$ ) and refrigerated storage (28 days). Samples were fermented with traditional yoghurt starters (Y; panel A) consisting of S. thermophilus ( $\mathbf{\Delta}$ ) and L. delbrueckii subsp. bulgaricus $(\triangle)$ compared with co-cultures of yoghurt starters with L. rhamnosus GG ( $\diamond$; Y-LGG, panel B) and B. animalis subsp. lactis BB12 ( $\bigcirc$; Y-BB12, panel C). Error bars represent standard deviations based on three independent replicates.

(Østlie, Helland, \& Narvhus, 2003). In association with yoghurt starters, the growth of $L$. rhamnosus GG was slightly enhanced, while the growth of $B$. animalis subsp. lactis BB12 was evidently stimulated. In comparison, the viable counts of L. rhamnosus GG and $B$. animalis subsp. lactis BB12 at the end of fermentation 


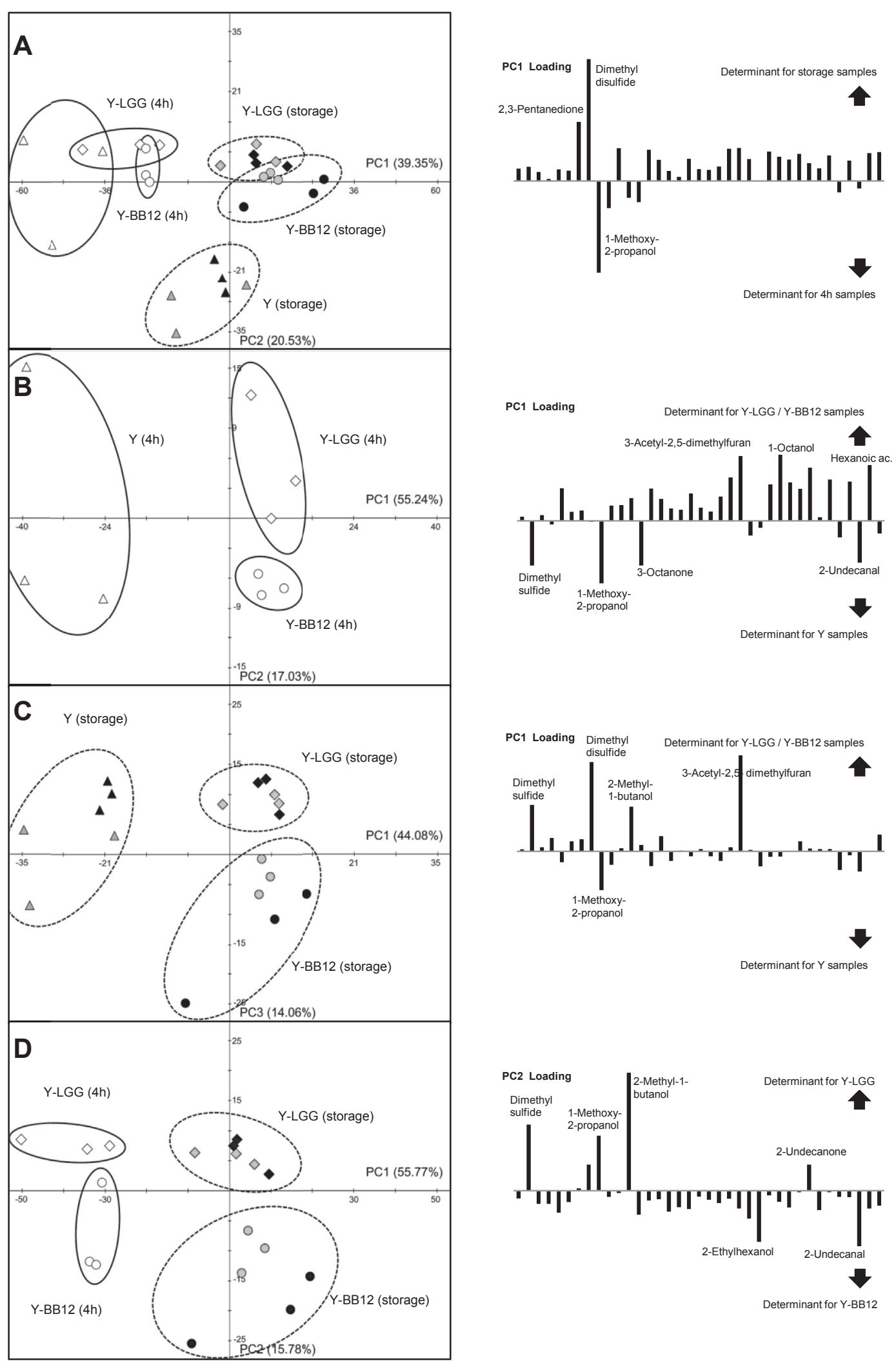

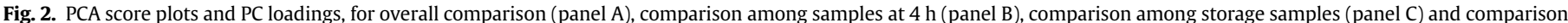

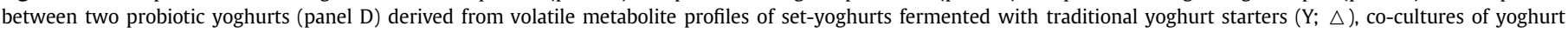

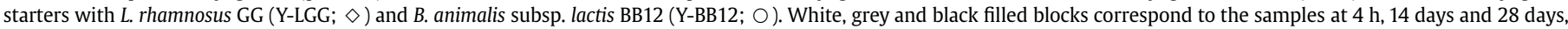
respectively. 
increased by 0.6 and $0.9 \log$ units to reach an average value of $6.7 \pm 0.1$ and $7.2 \pm 0.1 \log \mathrm{cfu} \mathrm{g}^{-1}$, respectively. This is in accordance with observations reported by El-Dieb, Abd Rabo, Badran, Abd ElFattah, and Elshaghabee (2012) and Saccaro et al. (2009), who also found an evident stimulatory effect on the growth of bifidobacteria in milk when co-cultivated with yoghurt starters. The viable counts of $L$. rhamnosus GG slightly decreased (0.5 log reduction) throughout the entire storage period while those of B. animalis subsp. lactis BB12 decreased (1.2 log reduction) especially during the first two weeks and then remained nearly stable until the end of storage. These results correspond with data from literature indicating a higher stability of probiotic lactobacilli compared with bifidobacteria in fermented milk (Gueimonde et al., 2004; Lourens-Hattingh \& Viljoen, 2001). The final viable counts of L. rhamnosus GG and B. animalis subsp. lactis BB12 at the end of storage reached an average value of $6.2 \pm 0.2$ and $6.1 \pm 0.2 \log \mathrm{cfu} \mathrm{g}^{-1}$, respectively, which still met the minimum recommended level (not less than $6.0 \mathrm{log} \mathrm{cfu} \mathrm{g}^{-1}$ ) to ensure their potential health-promoting effects (Shiby \& Mishra, 2013).

\subsection{Acidification profiles}

Similar acidification patterns were observed in all samples whether they were fermented with or without probiotics. At the end of fermentation, the $\mathrm{pH}$ values of all samples were not significantly different $(P>0.05)$ with an average value of $4.4 \pm 0.1$. The $\mathrm{pH}$ levels showed an evident decrease during storage especially during the first two weeks (ca. $0.3 \mathrm{pH}$ units); later, only a slight decline in $\mathrm{pH}$ was observed. At the end of storage, the $\mathrm{pH}$ values were reduced to an average of $4.1 \pm 0.1$ without significant difference $(P>0.05)$ due to the presence of probiotics.

To determine acid production, titratable acidity was measured during fermentation and storage expressed as millimoles $\mathrm{NaOH}$ per $100 \mathrm{~g}$ yoghurt (ISO/IDF, 2012). Higher acid production was found in the samples fermented with Y-LGG and Y-BB12 compared with those fermented with Y. At the end of fermentation, the titratable acidity detected in the samples of Y-LGG $\left(7.88 \mathrm{mmol} 100 \mathrm{~g}^{-1}\right)$ was slightly higher than that detected in the samples of Y-BB12 and Y (7.54 and $7.10 \mathrm{mmol} 100 \mathrm{~g}^{-1}$, respectively); however, the differences were not statistically significant $(P>0.05)$. A low capacity for acidifying milk by different probiotic strains was previously reported (Mohammadi et al., 2012; Sarkar, 2008). This finding contradicts with the study of Saccaro et al. (2009), who found a significant contribution of L. rhamnosus and B. animalis subsp. lactis to milk acidification when co-fermented with yoghurt starters. However, this might be related to differences in the strains used and other experimental factors, such as type and heating process of milk and fermentation conditions. At the end of storage, the titratable acidity detected in the samples of Y-LGG (12.10 mmol $100 \mathrm{~g}^{-1}$ ) was slightly higher than that detected in the samples of Y-BB12 and Y (11.32 and $10.43 \mathrm{mmol} 100 \mathrm{~g}^{-1}$, respectively). The difference in final titratable acidity between the samples of Y-LGG and Y was statistically significant $(P=0.03)$.

Decrease in $\mathrm{pH}$ and accumulation of organic acids during refrigerated storage of yoghurt are defined as "post-acidification", which is predominantly attributed to the metabolic activity of L. delbrueckii subsp. bulgaricus (Shah, 2000). This phenomenon has been identified as one of the most detrimental factors for the stability of probiotics during the shelf-life of yoghurt (Donkor et al., 2006). The results confirm a significant negative effect of postacidification on the viability of $L$. rhamnosus GG and $B$. animalis subsp. lactis BB12 in set yoghurt during refrigerated storage. Generally, lactobacilli are reported to be more tolerant to acidic condition of fermented milk than bifidobacteria (Donkor et al., 2006; El-Dieb et al., 2012).

\subsection{Volatile metabolite profiles determined by headspace SPME- GC/MS}

Volatile metabolite profiles of set yoghurts fermented with Y, YLGG and Y-BB12 were evaluated at the end of fermentation ( $4 \mathrm{~h})$ and every two weeks during storage ( $14 \mathrm{~d}$ and $28 \mathrm{~d}$ ). In this study, yoghurt samples were directly fermented in GC vials. The advantages of this approach are the small amount of sample required $(3 \mathrm{~mL})$ and minimal loss of volatiles during sample preparation (Settachaimongkon et al., 2014). To ensure that the results were comparable with the samples fermented in normal scale experiments $(100 \mathrm{~mL})$, the $\mathrm{pH}$ of samples fermented in GC vials was regularly verified. The results demonstrated no significant difference between the two approaches (data not shown). A total of 37 compounds comprising of alcohols, carbonyl compounds, organic acids, sulphur and heterocyclic compounds were identified in this study (Supplementary Table S1). This list is comparable with the volatiles identified in various types of yoghurt using SPME-GC/MS technique by others (Condurso, Verzera, Romeo, Ziino, \& Conte, 2008; Erkaya \& Sengul, 2011; Li, Zhang, \& Wang, 2013). However, the tightly sealed glass vial is impermeable and provides different protective properties compared with other packaging materials commonly used in yoghurt manufacture, e.g., laminated cartons, polypropylene, polystyrene and polyethylene (Tamime \& Robinson, 2007). The impact of packaging materials on aroma volatile compositions and sensory characteristics of yoghurt has been documented (Routray \& Mishra, 2011; Saint-Eve, Lévy, Le Moigne, Ducruet, \& Souchon, 2008). Therefore, this consideration needs to be taken into account when comparing the results in this research with those in practical situations.

PCA was performed to distinguish the profiles of volatile metabolites among set yoghurts fermented with Y, Y-LGG and Y-BB12. Samples at the end of fermentation $(4 \mathrm{~h})$ and during storage (14 d and $28 \mathrm{~d}$ ) with three replicates were statistically treated as individual objects $(n=27)$ in a multivariate analysis. A total of 37 volatile metabolites were introduced as variables. If necessary, missing values were replaced by the median of respective metabolites. An overall PCA score plot was constructed with a total variance of $59.9 \%$ (Fig. 2A). Within the group of $4 \mathrm{~h}$ samples, a good separation between Y and Y-BB12 was observed whereas the samples of Y-LGG overlapped with the two groups. The stored samples of $Y$ showed a well isolated volatile profile, while the stored samples of Y-LGG and Y-BB12 could not clearly be separated. Volatile metabolite profiles, of the $4 \mathrm{~h}$ samples could be distinguished from those of storage samples along PC1 (39.4\% variance). The metabolites accountable for separation are indicated in PC1loading. 1-Methoxy-2-propanol was a determinant for the $4 \mathrm{~h}$ samples, whereas the majority of volatiles, particularly dimethyl disulphide and 2,3-pentanedione, were accountable for the separation of stored samples.

To focus on the incorporation of probiotics, two PCA score plots were constructed for comparison among yoghurt samples at $4 \mathrm{~h}$ $(n=9)$ with a total variance of $72.3 \%$ (Fig. 2B) and among stored samples $(n=18)$ with a total variance of $58.2 \%$ (Fig. 2C). In both cases, volatile profiles of the samples fermented with Y were clearly separated from those fermented with Y-LGG and Y-BB12 along PC1 accounting for $55.2 \%$ and $44.1 \%$ variance, respectively. PC1-loading in Fig. 2B suggests that the activity of probiotics during fermentation facilitates the higher production of volatile metabolites in yoghurt. As storage time progressed, the numbers of metabolites contributing to separation decreased (PC1-loading in Fig. 2C). In other words, the overall metabolite composition of all samples became more similar to each other during storage. Despite low numbers of indicative metabolites in the loading plot, the stored samples of Y, Y-LGG and Y-BB12 were still completely separated. 
The final PCA score plot was constructed to evaluate the distinction between two probiotic strains (Fig. 2D), with a total variance of $71.6 \%$. The samples of Y-LGG and Y-BB12 were clearly separated along PC2, with metabolites accounting for separation indicated in the loading plot. These results demonstrate that volatile profiles of set yoghurts can successfully be distinguished according to the differences in types of starter cultures and also duration of storage. Besides this, indicative metabolites in the loading plots can be considered as potential biomarkers for detection of specific combinations of starter cultures and probiotics.

Heat-map visualisation combined with hierarchical cluster analysis was used to analyse the quantitative relationships of volatile profiles from different yoghurt samples (Fig. 3). Most metabolites are present in high relative abundances (shown in red or black) in the stored samples. The dendrogram shows that samples can be accurately grouped into different clusters, i.e., samples at $4 \mathrm{~h}$ of Y (A1), samples at $4 \mathrm{~h}$ of Y-LGG and Y-BB12 (A2), stored samples of Y (B) with an outlier from Y-BB12, stored samples of Y-LGG (C1) and stored samples of Y-BB12 (C2), according to their volatile profiles. This observation is in accordance with the PCA results in Fig. 2A, showing that volatile profiles of the samples fermented with Y-LGG and Y-BB12 are rather similar. However, unlike PCA, the overlap between two groups was not observed.

Acetaldehyde, diacetyl, acetoin, 2,3-pentanedione, acetone, 2butanone and acetic acid were present in high relative abundance in the samples (Fig. 4). These volatile metabolites are known as major aroma compounds of yoghurt (Cheng, 2010). Acetaldehyde (fresh, green, pungent) is the most important compound contributing to typical yoghurt aroma, which is mainly generated by threonine metabolism by yoghurt starters. Despite the high capacity for acetaldehyde production by $S$. thermophilus and L. delbrueckii subsp. bulgaricus (Beshkova, Simova, Frengova, \& Simov, 1998), bifidobacteria have also been reported to contribute to acetaldehyde formation in fermented milk (Baron, Roy, \& Vuillemard, 2000; Roy, 2005). This study confirmed that Y-BB12 resulted in significant higher acetaldehyde concentration $(P<0.05)$ in the samples at $4 \mathrm{~h}$ compared with Y and Y-LGG. Although this metabolite increased substantially during storage, there was no significant difference in acetaldehyde concentrations among yoghurt samples at the end of storage.

Diacetyl (buttery, creamy), acetoin (buttery) and 2,3pentanedione (buttery, vanilla-like) are primarily generated by S. thermophilus through pyruvate metabolism (Cheng, 2010). The results showed no significant difference in diacetyl and 2,3pentanedione levels among all yoghurt samples at $4 \mathrm{~h}$, while the level of acetoin was significantly higher $(P<0.05)$ in the samples fermented with Y-BB12. It has been reported that bifidobacteria may convert pyruvate to acetoin instead of organic acids to maintain their internal pH (Østlie et al., 2005).

Acetone and 2-butanone are naturally present in bovine milk (Hettinga et al., 2008), but a certain amount can be additionally produced by yoghurt starters (Cheng, 2010). The results showed that these two metabolites remained steady without any significant difference in either type of starter culture or storage duration. Despite the similar abundance in major aroma volatiles among yoghurt samples, most of the minor carbonyl compounds, volatile organic acids and alcohols were present in significantly higher abundance, especially in the samples co-fermented with probiotics (Supplementary Table S1). These metabolites also showed a significant increase during refrigerated storage. They may be generated from catabolism of pyruvate as well as amino acids or derived from other biochemical conversions by the activity of native milk enzymes remaining after pasteurisation, bacterial metabolism and lipid oxidation (Ardö, 2006; Beshkova et al., 1998; Urbach, 1995).

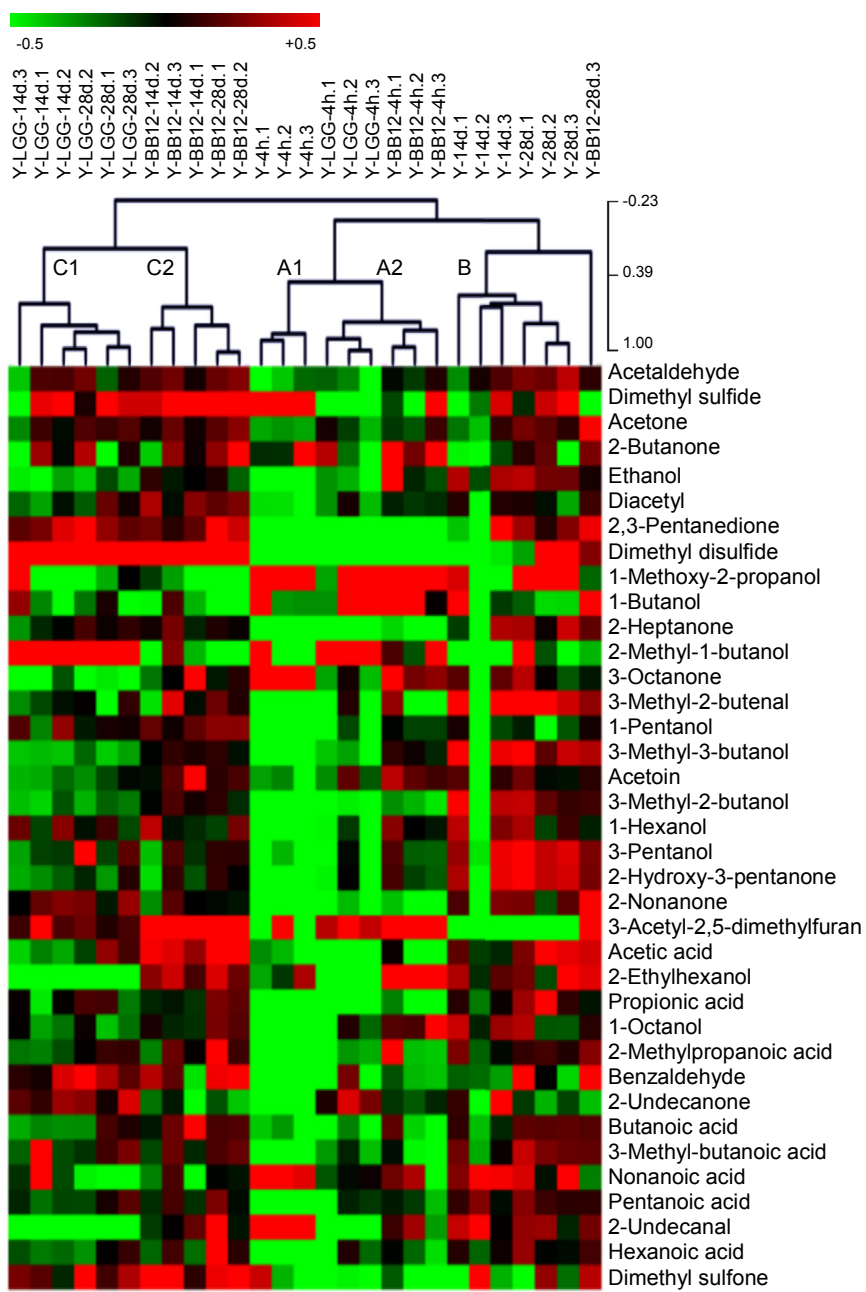

Fig. 3. Heat-map and hierarchical clustering of volatile metabolite profiles from setyoghurts fermented with traditional yoghurt starters (Y), co-cultures of yoghurt starters with L. rhamnosus GG (Y-LGG) and B. animalis subsp. lactis BB12 (Y-BB12). The dendrogram represents sample clusters based on Pearson's correlation coefficient with average linkage. Each square in the heat-map expresses normalised volatile content respected to the colour range. The red colour indicates higher content of the corresponding compound. For interpretation of the references to colour in this figure, the reader is referred to the web version of this article or Supplementary Material, Table S1.

The impact of L. rhamnosus GG and B. animalis subsp. lactis BB12 on benzaldehyde, organic acids and ethanol production in fermented milk has been previously reported (Østlie et al., 2003; Roy, 2005; Zareba, Ziarno, \& Obiedzinski, 2012). In particular, the capacity to convert lactose into acetic acid and lactic acid in the proportion of 3:2 (known as the Bifidus pathway) is a remarkable heterofermentative attribute of bifidobacteria (Tamime \& Robinson, 2007). However, the results did not show a significant increase in acetic acid concentration in the samples fermented with Y-BB12. The effects of high temperature incubation on the formation of acetic acid as well as other volatiles by bifidobacteria have been previously reported (Østlie et al., 2005). An incubation temperature near the optimum level for growth of bifidobacteria $\left(35-37^{\circ} \mathrm{C}\right)$ combined with a long incubation time $(10-18 \mathrm{~h}$ ) was found to be correlated with their capacity for volatile formation (Østlie et al., 2003; Zareba et al., 2012). Thus, a relatively high incubation temperature combined with rapid acidification rate by yoghurt starters in this study might explain the low production of acetic acid by B. animalis subsp. lactis BB12, although it was able to grow by approximately one $\log \mathrm{cfu} \mathrm{g}^{-1}$ during fermentation. Indeed, an 

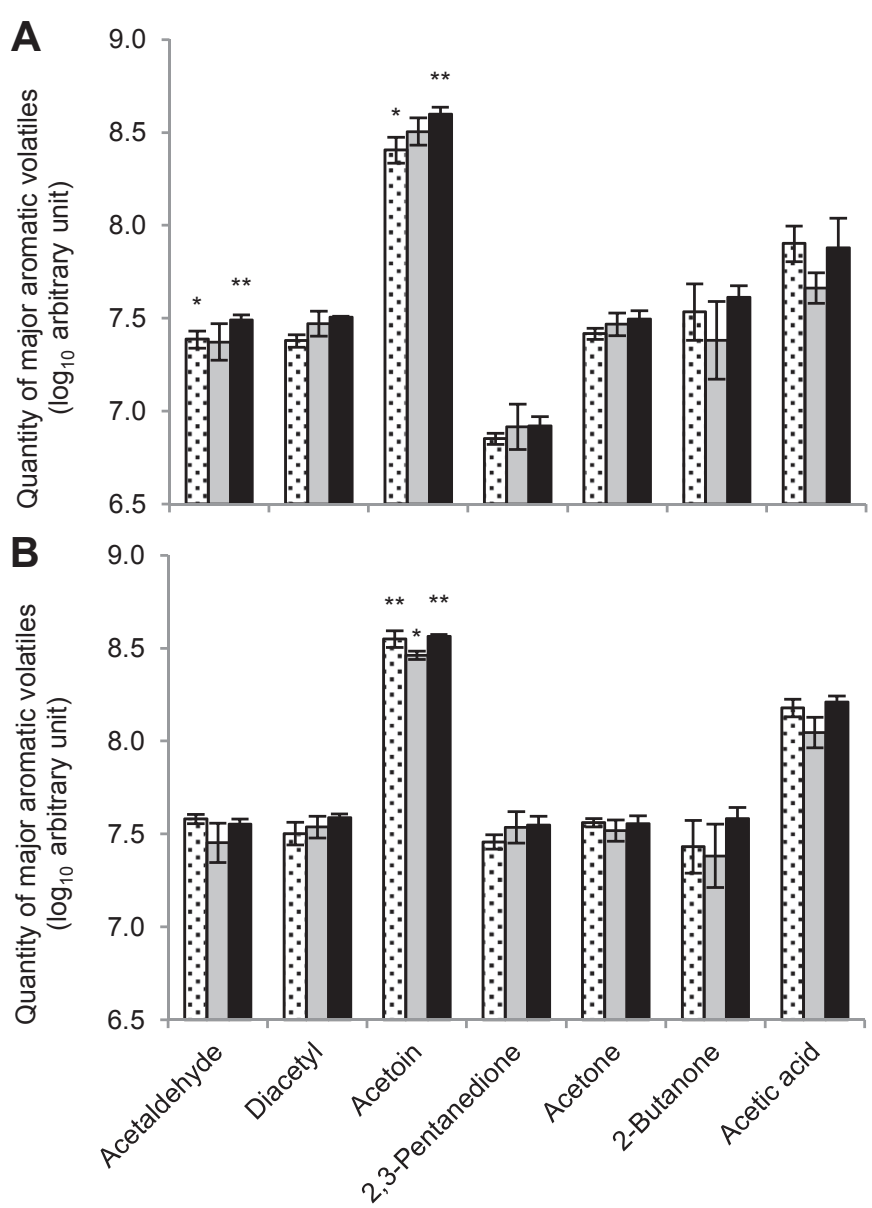

Fig. 4. Quantity of major volatile metabolites in set-yoghurt samples determined at the end of fermentation ( $4 \mathrm{~h}$; panel A) and the end of storage (28 days; panel B). Samples were fermented by traditional yoghurt starters (3) consisting of $S$. thermophilus and $L$. delbrueckii subsp. bulgaricus compared with co-culture of yoghurt starters with $L$. rhamnosus GG $(\square)$ and B. animalis subsp. lactis BB12 ( $\mathbf{\square})$. Error bars represent standard deviations based on three independent replicates. $\left(^{*}\right)$ indicates significant differences among mean values $(P<0.05)$ of samples.

excessive concentration of acetic acid (vinegar, pungent) in yoghurt may be considered as undesirable.

In summary, the results demonstrate that the incorporation of L. rhamnosus GG and B. animalis subsp. lactis BB12 did not significantly influence the major aroma volatile metabolites desirable for a good organoleptic quality of yoghurt. However, contributions of L. rhamnosus GG and B. animalis subsp. lactis BB12 are likely pronounced in the formation of minor volatile metabolites present at low concentration, especially volatile organic acids and alcohols, in yoghurt. This information is technologically relevant, since variations in these compounds may also influence the organoleptic quality of products. From a short term perspective, the absolute concentration of volatile metabolites should be quantified. This will provide more insight whether the amount of these compounds is detected within the same ranges as normally found in literature or commercial products. Based on this information, the potential impact on the organoleptic quality of yoghurt could be appropriately predicted.

\subsection{Non-volatile polar metabolite profiles determined by ${ }^{1} \mathrm{H} N \mathrm{NR}$}

A total of 43 metabolites, including amino acids, carbohydrates, organic acids, lipid derivatives, carbonyl compounds, one sulphur compound and one nucleoside were identified based on the list in our previous study (Settachaimongkon et al., 2014). The quantification was performed by summation of signal intensities in all bins corresponding to the target metabolite (Park et al., 2013). The integrated intensities were finally expressed in $\log _{10}$ arbitrary unit (Supplementary Table S2). For multivariate analysis, it should be mentioned that the 43 identified metabolites accounted for labelling of 149 bins. A complementary data filtering by ANOVA was performed for selection of the remaining unknowns (Lamanna, Braca, Di Paolo, \& Imparato, 2011). Finally, a total of 214 bins were introduced as variables for the analyses.

An overall PCA score plot was constructed with a total variance of $65.5 \%$ (Fig. 5A). The distinction among all the $4 \mathrm{~h}$ samples was rather small but they could still be separated. It was evident that non-volatile profiles of the samples at $4 \mathrm{~h}$ could be distinguished from those of stored samples along PC1 (36.1\% variance). The loading plot indicates that the majority of metabolites in the sugar region are determinant for the samples at $4 \mathrm{~h}$, whereas those from amino acids and lactate contribute to the separation of stored samples. To focus on the incorporation of probiotics, two separate PCA score plots were constructed for comparison among yoghurt samples at $4 \mathrm{~h}(n=6)$, with a total variance of $73.8 \%$ (Fig. 5B), and among stored samples $(n=6)$, with a total variance of $83.4 \%$ (Fig. 5C). In both cases, the samples fermented with Y, Y-LGG and YBB12 were clearly separated from each other. It was remarkable that the distinction is larger among stored samples. For instance, a complete separation was found between the samples of Y-LGG and those of $\mathrm{Y}$ and $\mathrm{Y}-\mathrm{BB} 12$, as determined by formate, pyruvate, oxoglutarate, fumarate and uridine along PC1. These metabolites provide a good indication for substantial metabolic activity of L. rhamnosus GG contributing to the changes in non-volatile metabolite profile of set yoghurt during refrigerated storage. The results demonstrate that non-volatile polar metabolite profiles of set yoghurts can successfully be distinguished according to the differences in types of starter cultures and also duration of storage.

Heat-map visualisation combined with hierarchical cluster analysis was used to analyse the quantitative relationships of nonvolatile polar metabolite profiles of different samples (Fig. S1). The results illustrated on-going metabolic activity of starter cultures under refrigerated conditions, i.e., amino acids and organic acids were detected in high abundance while metabolites in the sugar group notably decreased. As storage time progressed, Pearson's correlation-based linkages showed that non-volatile metabolite profiles of the samples fermented with Y-LGG were well separated. This observation suggests a contribution of the activity of L. rhamnosus GG to the formation of non-volatile metabolites in set yoghurt during refrigerated storage.

During fermentation, the role of $S$. thermophilus and L. delbrueckii subsp. bulgaricus in the biochemical conversion of milk components has well been documented (Tamime \& Robinson, 2007). The proto-cooperation between these two species, based on the exchange of several metabolically derived compounds that provide mutual growth stimulating effects to each other (Sieuwerts, de Bok, Hugenholtz, \& van Hylckama Vlieg, 2008), leads to the production of various metabolites, resulting in a formation of semi-solid texture and typical yoghurt flavour (Courtin \& Rul, 2004). The primary role of dairy starter cultures is acidification of milk by conversion of lactose into lactic acid. Although individual cultures of $L$. rhamnosus GG and B. animalis subsp. lactis BB12 exhibit low acidifying capacity in milk, their contribution to acidification may increase from slight to a significant level in co-culture with yoghurt starters (Roy, 2005; Sarkar, 2008). According to the quantification of non-volatile metabolites presented in Supplementary Table S2, the results confirmed that lactate concentration was slightly higher in the samples fermented with YLGG and Y-BB12 compared with those fermented without 

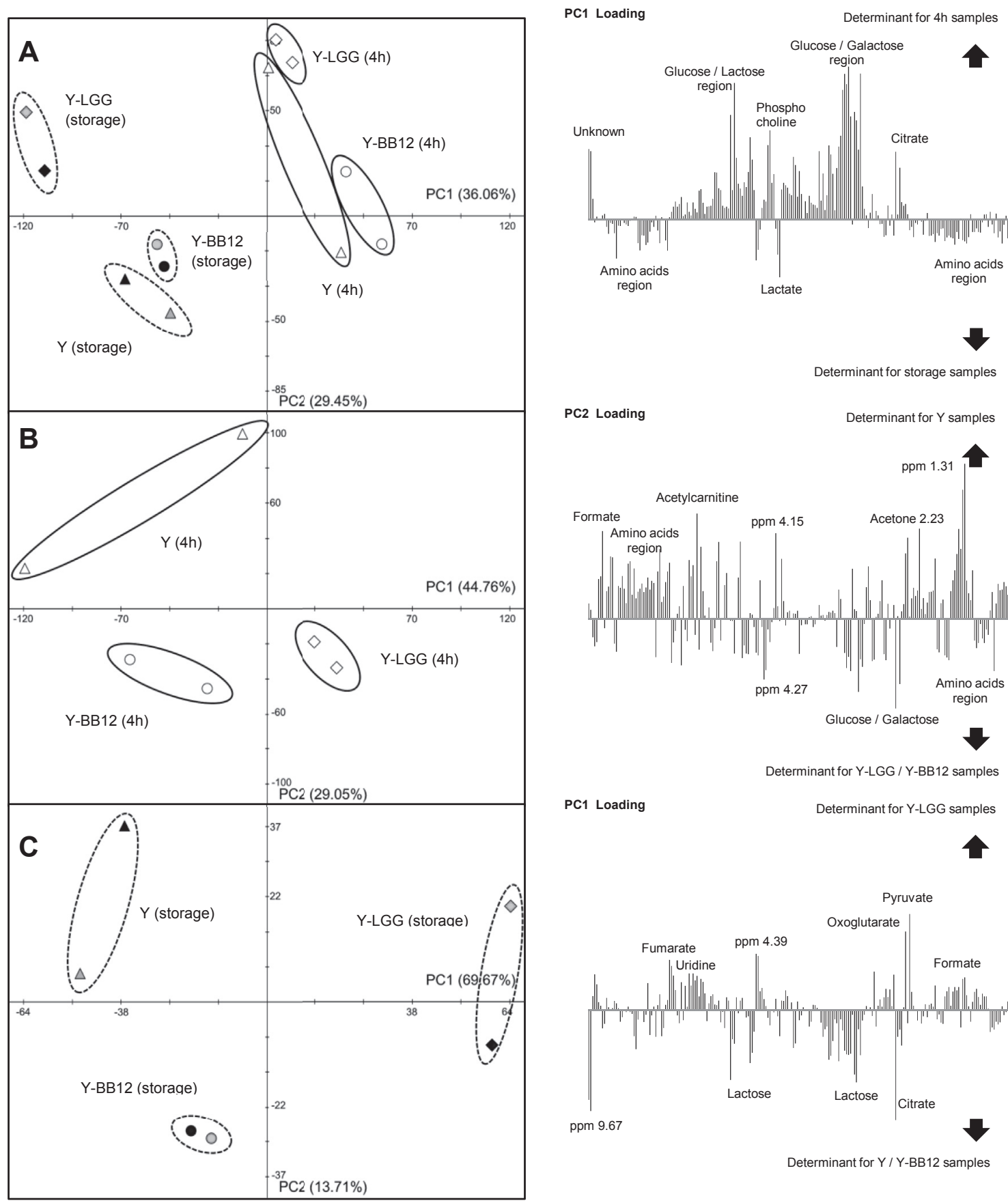

PC1 Loading Determinant for Y-LGG samples

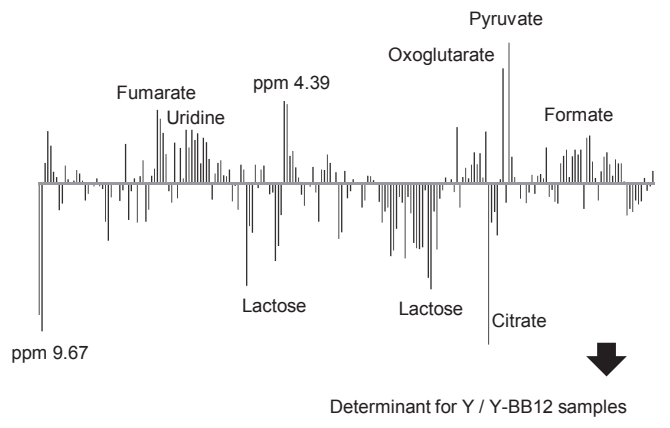

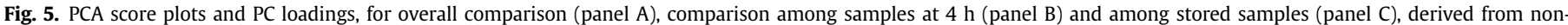

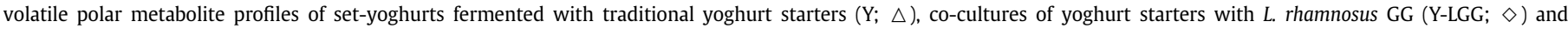
B. animalis subsp. lactis BB12 (Y-BB12; O). White, grey and black filled blocks correspond to the samples at $4 \mathrm{~h}, 14$ days and 28 days, respectively.

probiotics. Due to the lack of ability to ferment lactose of L. rhamnosus GG, this result suggests that this strain might take advantage from the free galactose generated by $\beta$-galactosidase activity of yoghurt starters.

In contrast to the homofermentative yoghurt starters, L. rhamnosus GG and B. animalis subsp. lactis BB12 are classified as heterofermentative, i.e., certain amounts of lactate, acetate, ethanol and $\mathrm{CO}_{2}$ can be simultaneously generated from their carbohydrate metabolism (Tamime \& Robinson, 2007). Therefore, an increase in these compounds during fermentation could directly indicate the activity of probiotics. This was confirmed by higher amounts of acetate and ethanol (previously mentioned) detected in the samples fermented with Y-LGG and Y-BB12. Nevertheless, the difference in acetic acid concentration was negligible in their volatile profiles. Ascorbate, isobutyrate and succinate were present at higher concentrations especially in the samples fermented with YBB12. However, contributions by these organic acids were rather small and did not contribute to a significant difference in acidification profiles among yoghurt samples. Pyruvate is a key metabolite derived from carbohydrate metabolism and can be further converted into various organic acids and volatiles (Walstra, Wouters, \& Geurts, 2006). This metabolite was evidently more 
abundant in the samples fermented with Y-LGG and Y-BB12. The influence of proteolytic activity was characterised by an overall increase in free amino acid concentrations. Growth of bifidobacteria in milk is restricted due to their low proteolytic activity (Roy, 2005). However, active growth of B. animalis subsp. lactis BB12 in co-culture with yoghurt starters found in this study suggests that bifidobacteria might take advantage of free nitrogen sources supplied by the proteolytic activity of $L$. delbrueckii subsp. bulgaricus. A small increase in free amino acid concentrations was observed in the samples of Y-BB12, particularly for alanine, phenylalanine, tyrosine and valine.

During refrigerated storage, decreases in lactose and glucose were observed in all samples and corresponded with an increase in lactate concentration. Increases in other organic acids and free amino acids were clearly observed in the samples fermented with Y-LGG. It is possible that proteolysis-derived compounds increased the buffering capacity of milk. This could explain the significant difference in titratable acidy observed between the samples of $\mathrm{Y}$ and Y-LGG without significant difference in $\mathrm{pH}$ value. The results indicate a substantial metabolic activity of YLGG during refrigerated storage, which corresponds with a distinctive non-volatile polar metabolite profile, as demonstrated by multivariate analysis.

\section{Conclusions}

A complementary metabolomics approach using headspace SPME-GC/MS and ${ }^{1} \mathrm{H}$ NMR was used for characterisation of volatile and non-volatile polar metabolite profiles of set yoghurt during fermentation and storage. L. rhamnosus GG and B. animalis subsp. lactis BB12 did not influence acidity and major aroma volatile metabolites desirable for a good organoleptic quality of yoghurt. On the other hand, a contribution of $L$. rhamnosus GG to non-volatile polar metabolite profile of yoghurt was seen during refrigerated storage. The combination of metabolomic-derived data with multivariate analysis allows discrimination of yoghurt samples statistically according to the difference in types of starter combinations, together with durations of storage. This finding provides new insights regarding the impact of probiotics on the metabolome of yoghurt.

\section{Acknowledgements}

This research was conducted under CHE-PhD-SFR-2551 scholarship granted by the Commission on Higher Education of the Royal Thai Government. Yoghurt starters were kindly provided by Bert Hafkamp from CSK Food Enrichment. The authors are grateful to Dr. Kasper Hettinga, Geert Meijer and Dr. Jacques Vervoort for assistance in GC/MS and NMR analysis. We also thank Vera Winata, Xiaoxi Wang, Anastasia Emelianova and $\mathrm{Wu} \mathrm{Qu}$ for technical contributions.

\section{Appendix A. Supplementary data}

Supplementary data related to this article can be found at http:// dx.doi.org/10.1016/j.idairyj.2014.04.002.

\section{References}

Ardö, Y. (2006). Flavour formation by amino acid catabolism. Biotechnology Advances, $24,238-242$.

Ashraf, R., \& Shah, N. P. (2011). Selective and differential enumerations of Lactobacillus delbrueckii subsp. bulgaricus, Streptococcus thermophilus, Lactobacillus acidophilus, Lactobacillus casei and Bifidobacterium spp. in yoghurt - a review. International Journal of Food Microbiology, 149, 194-208.
Baron, M., Roy, D., \& Vuillemard, J. C. (2000). Biochemical characteristics of fermented milk produced by mixed-cultures of lactic starters and bifidobacteria. Lait, 80, 465-478.

Beshkova, D., Simova, E., Frengova, G., \& Simov, Z. (1998). Production of flavour compounds by yogurt starter cultures. Journal of Industrial Microbiology and Biotechnology, 20, 180-186.

Boudonck, K., Mitchell, M., Wulff, J., \& Ryals, J. (2009). Characterization of the biochemical variability of bovine milk using metabolomics. Metabolomics, 5, 375-386.

Cheng, H. (2010). Volatile flavor compounds in yogurt: a review. Critical Reviews in Food Science and Nutrition, 50, 938-950.

Codex. (2003). CODEX STAN 243-2003: Standard for fermented milks (revised in 2010 ed., Vol. 2012) http://www.fao.org/docrep/015/i2085e/i2085e00.pdf.

Condurso, C., Verzera, A., Romeo, V., Ziino, M., \& Conte, F. (2008). Solid-phase microextraction and gas chromatography mass spectrometry analysis of dairy product volatiles for the determination of shelf-life. International Dairy Journal, $18,819-825$.

Consonni, R., \& Cagliani, L. R. (2008). Ripening and geographical characterization of Parmigiano Reggiano cheese by 1H-NMR spectroscopy. Talanta, 76, 200-205.

Courtin, P., \& Rul, F. (2004). Interactions between microorganisms in a simple ecosystem: yogurt bacteria as a study model. Lait, 84, 125-134.

Donkor, O. N., Henriksson, A., Vasiljevic, T., \& Shah, N. P. (2006). Effect of acidification on the activity of probiotics in yoghurt during cold storage. International Dairy Journal, 16, 1181-1189.

El-Dieb, S. M., Abd Rabo, F. H. R., Badran, S. M., Abd El-Fattah, A. M., \& Elshaghabee, F. M. F. (2012). The growth behaviour and enhancement of probiotic viability in bioyoghurt. International Dairy Journal, 22, 44-47.

Erkaya, T., \& Sengul, M. (2011). Comparison of volatile compounds in yoghurts made from cows', buffaloes', ewes' and goats' milks. International Journal of Dairy Technology, 64, 240-246.

FAO/WHO. (2002). Guidelines for the evaluation of probiotics in food. Report of a joint FAO (Food and Agriculture Organization of the United Nationas)/WHO (World Health Organization) working group on drafting guidelines for the evaluation of probiotics in food. London Ontario, Canada: FAO.

Gueimonde, M., Delgado, S., Mayo, B., Ruas-Madiedo, P., Margolles, A., \& de los Reyes-Gavilán, C. G. (2004). Viability and diversity of probiotic Lactobacillus and Bifidobacterium populations included in commercial fermented milks. Food Research International, 37, 839-850.

Herrero, M., Simo, C., Garcia-Canas, V., Ibanez, E., \& Cifuentes, A. (2012). Foodomics: MS-based strategies in modern food science and nutrition. Mass Spectrometry Reviews, 31, 49-69.

Hettinga, K. A., van Valenberg, H. J. F., Lam, T. J. G. M., \& van Hooijdonk, A. C. M. (2008). Detection of mastitis pathogens by analysis of volatile bacterial metabolites. Journal of Dairy Science, 91, 3834-3839.

Hettinga, K. A., van Valenberg, H. J. F., Lam, T. J. G. M., \& van Hooijdonk, A. C. M. (2009). The origin of the volatile metabolites found in mastitis milk. Veterinary Microbiology, 137, 384-387.

ISO/IDF. (2012). ISO/TS11869-IDF/RM150:2012-Fermented milks - determination of titratable acidity - potentiometric method. Geneva, Switzerland: International Organisation for Standardisation.

Klein, M. S., Almstetter, M. F., Schlamberger, G., Nürnberger, N., Dettmer, K., Oefner, P. J., et al. (2010). Nuclear magnetic resonance and mass spectrometrybased milk metabolomics in dairy cows during early and late lactation. Journal of Dairy Science, 93, 1539-1550.

Lamanna, R., Braca, A., Di Paolo, E., \& Imparato, G. (2011). Identification of milk mixtures by $1 \mathrm{H}-\mathrm{NMR}$ profiling. Magnetic Resonance in Chemistry, 49, S22-S26.

Li, Y., Zhang, L., \& Wang, W. (2013). Heat-induced changes in volatiles of milk and effects of thermal processing on microbial metabolism of yogurt. Journal of Food Biochemistry, 37, 409-417.

Lourens-Hattingh, A., \& Viljoen, B. C. (2001). Yogurt as probiotic carrier food. International Dairy Journal, 11, 1-17.

Lu, J., Antunes Fernandes, E., Paez Cano, A. E., Vinitwatanakhun, J., Boeren, S., van Hooijdonk, T., et al. (2013). Changes in milk proteome and metabolome associated with dry period length, energy balance, and lactation stage in postparturient dairy cows. Journal of Proteome Research, 12, 3288-3296.

Mohammadi, R., Sohrabvandi, S., \& Mohammad-Mortazavian, A. (2012). The starter culture characteristics of probiotic microorganisms in fermented milks. Engineering in Life Sciences, 12, 399-409.

Mozzi, F., Ortiz, M. E., Bleckwedel, J., De Vuyst, L., \& Pescuma, M. (2013). Metabolomics as a tool for the comprehensive understanding of fermented and functional foods with lactic acid bacteria. Food Research International, 54, 11521161.

Oliveira, M. N., Sodini, I., Remeuf, R., Tissier, J. P., \& Corrieu, G. (2002). Manufacture of fermented lactic beverages containing probiotic cultures. Journal of Food Science, 67, 2336-2341.

Østlie, H. M., Helland, M. H., \& Narvhus, J. A. (2003). Growth and metabolism of selected strains of probiotic bacteria in milk. International Journal of Food Microbiology, 87, 17-27.

Østlie, H. M., Treimo, J., \& Narvhus, J. A. (2005). Effect of temperature on growth and metabolism of probiotic bacteria in milk. International Dairy Journal, 15, 989997.

Park, S. J., Hyun, S. H., Suh, H. W., Lee, S. Y., Sung, G. H., Kim, S. H., et al. (2013). Biochemical characterization of cultivated Cordyceps bassiana mycelia and fruiting bodies by $1 \mathrm{H}$-nuclear magnetic resonance spectroscopy. Metabolomics, 9, 236-246. 
Piras, C., Cesare Marincola, F., Savorani, F., Engelsen, S. B., Cosentino, S., Viale, S., et al. (2013). A NMR metabolomics study of the ripening process of the Fiore Sardo cheese produced with autochthonous adjunct cultures. Food Chemistry, 141, 2137-2147.

Plessas, S., Bosnea, L., Alexopoulos, A., \& Bezirtzoglou, E. (2012). Potential effects of probiotics in cheese and yogurt production: a review. Engineering in Life Sciences, $12,433-440$.

Rodrigues, D., Santos, C. H., Rocha-Santos, T. A. P., Gomes, A. M., Goodfellow, B. J., \& Freitas, A. C. (2011). Metabolic profiling of potential probiotic or synbiotic cheeses by nuclear magnetic resonance (NMR) spectroscopy. Journal of Agricultural and Food Chemistry, 59, 4955-4961.

Routray, W., \& Mishra, H. N. (2011). Scientific and technical aspects of yogurt aroma and taste: a review. Comprehensive Reviews in Food Science and Food Safety, 10, $208-220$.

Roy, D. (2005). Technological aspects related to the use of bifidobacteria in dairy products, Lait, 85, 39-56.

Saccaro, D. M., Tamime, A. Y., Pilleggi, A. L. O. P. S., \& Oliveira, M. N. (2009). The viability of three probiotic organisms grown with yoghurt starter cultures during storage for 21 days at $4{ }^{\circ} \mathrm{C}$. International Journal of Dairy Technology, 62, 397-404.

Saint-Eve, A., Lévy, C., Le Moigne, M., Ducruet, V., \& Souchon, I. (2008). Quality changes in yogurt during storage in different packaging materials. Food Chemistry, 110, 285-293.

Sarkar, S. (2008). Effect of probiotics on biotechnological characteristics of yoghurt: a review. British Food Journal, 110, 717-740.

Settachaimongkon, S., Nout, M. J. R., Antunes Fernandes, E. C., Hettinga, K. A., Vervoort, J. M., van Hooijdonk, T. C. M., et al. (2014). Influence of different proteolytic strains of Streptococcus thermophilus in co-culture with Lactobacillus delbrueckii subsp. bulgaricus on the metabolite profile of set yoghurt. International Journal of Food Microbiology, 177, 29-36.

Shah, N. P. (2000). Probiotic bacteria: selective enumeration and survival in dairy foods. Journal of Dairy Science, 83, 894-907.

Shiby, V. K., \& Mishra, H. N. (2013). Fermented milks and milk products as functional foods: a review. Critical Reviews in Food Science and Nutrition, 53, 482496.

Sieuwerts, S., de Bok, F. A. M., Hugenholtz, J., \& van Hylckama Vlieg, J. E. T. (2008). Unraveling microbial interactions in food fermentations: from classical to genomics approaches. Applied and Environmental Microbiology, 74, 4997-5007.

Tamime, A. Y., \& Robinson, R. K. (2007). Tamime and Robinson's yoghurt: Science and technology (3rd ed.). Cambridge, UK: CRC Press.

Tharmaraj, N., \& Shah, N. P. (2003). Selective enumeration of Lactobacillus delbrueckii ssp. bulgaricus, Streptococcus thermophilus, Lactobacillus acidophilus, Bifidobacteria, Lactobacillus casei, Lactobacillus rhamnosus, and Propionibacteria. Journal of Dairy Science, 86, 2288-2296.

Urbach, G. (1995). Contribution of lactic acid bacteria to flavour compound formation in dairy products. International Dairy Journal, 5, 877-903.

Vasiljevic, T., \& Shah, N. P. (2008). Probiotics: from Metchnikoff to bioactives. International Dairy Journal, 18, 714-728.

Walstra, P., Wouters, J. T. M., \& Geurts, T. J. (2006). Dairy science and technology (2nd ed.). New York, NY, USA: CRC/Taylor \& Francis.

Zareba, D., Ziarno, M., \& Obiedzinski, M. (2012). Volatile profile of non-fermented milk and milk fermented by Bifidobacterium animalis subsp. lactis. International Journal of Food Properties, 15, 1010-1021. 